\title{
Asymptotic analysis of the dewetting rim
}

\author{
Jacco H. Snoeijer ${ }^{1}$ and Jens Eggers ${ }^{2}$ \\ ${ }^{1}$ Physics of Fluids Group and J. M. Burgers Centre for Fluid Dynamics, University of Twente, P.O. Box 217, 7500 AE Enschede, \\ The Netherlands \\ ${ }^{2}$ Department of Mathematics, University of Bristol, University Walk, Bristol BS8 1TW, United Kingdom \\ (Received 21 May 2010; revised manuscript received 21 September 2010; published 15 November 2010)
}

\begin{abstract}
Consider a film of viscous liquid covering a solid surface, which it does not wet. If there is an initial hole in the film, the film will retract further, forming a rim of fluid at the receding front. We calculate the shape of the rim as well as the speed of the front using lubrication theory. We employ asymptotic matching between the contact line region, the rim, and the film. Our results are consistent with simple ideas involving dynamic contact angles and permit us to calculate all free parameters of this description, previously unknown.
\end{abstract}

DOI: 10.1103/PhysRevE.82.056314

PACS number(s): 47.55.nb, 68.08.Bc

\section{INTRODUCTION}

The dynamics by which a surface, previously covered by a fluid film, dries up is of fundamental importance $[1,2]$. The driving force for the dynamics of drying is provided by surface energy. If the equilibrium contact angle $\theta_{e q}$ between the fluid and the substrate is greater than zero (or the spreading coefficient is negative [2]), the dry solid is energetically favored over one covered by a macroscopic film. However, for the system to harvest this energy, an initially dry region has to be produced.

A lot of attention has focused recently on the case of ultrathin films [3-6], whose thickness is in the range of a few nanometers. For them to become unstable, intermolecular forces have to be considered [7]. In addition, it has been argued that slip [8] or non-Newtonian effects $[9,10]$ may be important for describing the film profile, even away from the contact line. However, macroscopic films are equally relevant, into which a hole can be made either mechanically [11] or by instability, driven, e.g. by evaporation.

We focus exclusively on the rapid dynamics that ensues and on Newtonian fluid dynamics dominated by viscosity, as is usual for thin films. The phenomenology of the dewetting process can be summarized as follows $[1,12]$. As the contact line bordering the film retracts over the solid, the liquid inside the film is collected into a rim, which grows slowly in time, as sketched in Fig. 1. The height and half-width of the rim are denoted by $h_{r}$ and $w$, respectively, and will become large compared to the film thickness $h_{f}$. Even though the problem may be axisymmetric initially (a circular hole), the radius of this hole is soon much larger than the rim, in which case the contact line may be considered straight and the problem becomes two dimensional. It was found experimentally that the speed of retraction $U$ of a viscous film is constant [12]. The goal is to identify $U$ and to compute the shape of the rim, characterized mainly by the associated apparent contact angle $\theta_{a p}$ (cf. Fig. 1).

The first experiments indeed reported a constant speed of dewetting, which depends on the equilibrium contact angle as $U \sim \theta_{e q}^{3}[12]$, which is a scaling common for wetting dynamics. The apparent contact angle was found to be $\theta_{a p} / \theta_{e q} \approx 0.7$. However, a later study [11] found a much smaller value of $\theta_{a p} / \theta_{e q} \approx 0.15$. This is troubling since the value of the apparent angle is very important for the selection of the speed. As described, e.g., in [1], the problem consists essentially of the dynamics of a receding front at the contact line (forming the front of the rim), coupled to an advancing front (forming the back of the rim). The dewetting speed is determined by equating $\theta_{a p}$ for both fronts. Note that most wetting problems are dominated by an isolated advancing or receding contact line, in which case no unique speed can be identified [13].

The present problem has been analyzed before $[1,12,14-16]$, but the required matching procedure has never been carried through. Indeed, we identify an error present in many of the earlier treatments $[1,12,14,15]$, which are based on a simplified description of moving contact lines developed in [17]. The observation underlying the present paper is that the entire structure of both fronts, including the quasistatic central part of the rim, can be described by a single equation in the lubrication limit. Moreover, this equation possesses an exact solution [18], which simplifies the analysis tremendously and makes the calculation of all required constants feasible.

\section{PROBLEM FORMULATION}

We treat the profile as two dimensional, assuming that the size of the hole is large with respect to the rim. For small contact angles, $\theta_{e q} \ll 1$, the interface profile $h(x, t)$ can be computed from the two-dimensional lubrication approximation in the frame comoving with the contact line [2]:

$$
\partial_{t} h-U \partial_{x} h+\frac{\gamma}{3 \eta} \partial_{x}\left[h^{2}(h+3 \lambda) \partial_{x x x} h\right]=0,
$$

where $\eta$ and $\gamma$ denote viscosity and surface tension, respectively, while $U$ is the speed of dewetting. We further intro-

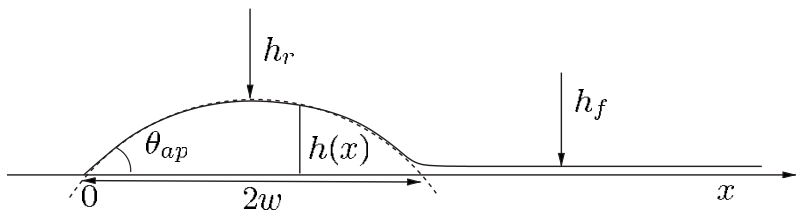

FIG. 1. Sketch of the dewetting rim profile $h(x)$. A liquid film of thickness $h_{f}$ is invaded by a moving rim of height $h_{r}$ and width $w$. The apparent contact angle $\theta_{a p}$ is defined as the intersection of the solid surface and a parabolic fit of the rim (dashed line). 
duced a slip length $\lambda$, necessary to relieve the moving contact line singularity $[2,19]$. This quantifies the scale, typically a few molecular sizes, at which the no-slip boundary is violated. Boundary conditions at the contact line at $x=0$ are

$$
\begin{gathered}
h(0, t)=0, \\
\partial_{x} h(0, t)=\theta_{e q},
\end{gathered}
$$

while on the other side of the rim, the profile should approach the thickness of the prewetted film,

$$
h(x \rightarrow \infty, t)=h_{f} .
$$

Since the rim grows in time, the solution is intrinsically time dependent. Based on volume conservation, however, one finds that there is a separation of time scales between the growth of the rim and the dynamics of retraction. Thus, the problem can be treated as quasisteady. Namely, the area of the rim is of order $A(t) \sim h_{r} w$, and thus grows at a rate $\dot{A}$ $\sim h_{r} \dot{w}$. This growth is due to liquid inside the film that is swallowed by the advancing rim, occurring at a rate $\sim U h_{f}$ (Fig. 1). Hence, one finds

$$
\frac{\dot{w}}{U} \sim \frac{h_{f}}{h_{r}},
$$

which becomes asymptotically small in the long-time limit. This illustrates that changes in the geometry of the rim are slow with respect to $U$, allowing us to drop $\partial_{t} h$ in the lubrication equation (1). Integrating once, Eq. (1) takes the form

$$
-\mathrm{Ca} h+\frac{1}{3} h^{2}(h+3 \lambda) \partial_{x x x} h=Q,
$$

where we introduce the capillary number $\mathrm{Ca}=U \eta / \gamma$ and $Q$ is a constant of integration representing the flux of liquid (volume per unit time per unit length). We note that within the lubrication approximation both $\theta_{e q}$ and $\mathrm{Ca}^{1 / 3}$ are small and of similar magnitude.

Note that form (6) appears to be inconsistent with the boundary conditions of the problem. On one hand, the contact line naturally gives a vanishing flux, $Q_{\mathrm{cl}}=0$, since $h=0$. By contrast, one finds $\partial_{x x x} h=0$ in the film region, yielding $Q_{f}=-\mathrm{Ca} h_{f}$. This apparent inconsistency can be traced back to the slow dynamics of the rim, making the steady ansatz not an exact solution of the problem. Rather, terms of order $h_{f} / h_{r}$ have been neglected, as implied by Eq. (5).

The strategy of our analysis is to treat the domains near the front and the back of the rim separately and match their asymptotic behaviors. We take the respective values of $Q$ explained above and define $h(x)$ by

$$
\begin{aligned}
& h^{\prime \prime \prime}=\frac{3 \mathrm{Ca}}{h(h+3 \lambda)}, \quad \text { for } 0 \leq x<x^{*}, \\
& h^{\prime \prime \prime}=\frac{3 \mathrm{Ca}}{h^{2}}\left(1-\frac{h_{f}}{h}\right), \quad \text { for } x^{*}<x,
\end{aligned}
$$

with boundary conditions (2)-(4). The solutions of the two equations should be matched in an overlap region around $x^{*}$ where the thickness $h$ is sufficiently large for Eqs. (7) and (8) to be identical. Note that in deriving Eq. (8) we have also

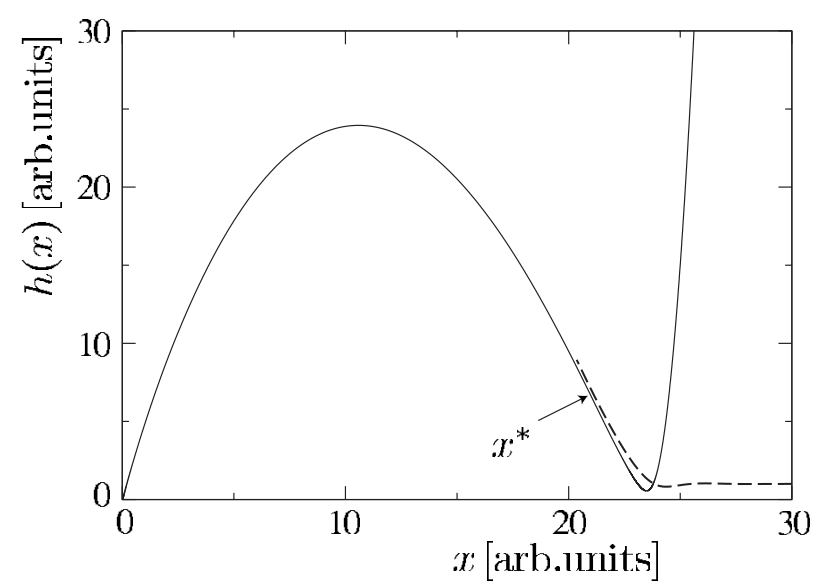

FIG. 2. Schematic representation of the matching procedure. The contact line and rim are described by the solid line, from Eq. (7). The dashed line represents the profile in the film region, from Eq. (8). The profiles are matched at the advancing side of rim around $x^{*}$.

taken the film thickness to be much larger than the slip length. In summary, the problem can be characterized by the relations

$$
\lambda \ll h_{f} \ll h_{r} \sim \theta_{e q} w .
$$

This simplifies the problem since both equations now contain only a single length scale.

\section{MATCHING}

The strategy of our analysis is outlined in Fig. 2. The profile of the contact line and rim, described by Eq. (7), can be solved analytically and is shown as the solid line. Owing to this solution one avoids having to match the receding contact line to a rim of negative curvature, which is a subtle problem [13]. This solution does not connect to the film, but instead reaches a minimum value and behaves as $\sim x^{2}$ for large $x$. However, the intermediate asymptotics just before the minimum is reached (around $x^{*}$ ) can be matched to the solution of Eq. (8), which is shown as the dashed line. Below we work out the asymptotic expansions and find the speed of the dewetting rim from the matching.

\section{A. Contact line and rim solution}

The only length scale appearing in Eq. (7) is the slip length $\lambda$, suggesting a rescaling

$$
h(x)=3 \lambda H\left(\frac{x \theta_{e q}}{3 \lambda}\right), \quad \xi=\frac{x \theta_{e q}}{3 \lambda} .
$$

Here, we have chosen the horizontal and vertical scales to differ by a factor $\theta_{e q}$, which ensures the boundary condition $H^{\prime}(0)=1$. Inserting this scaling into Eq. (7) provides the equation for the dimensionless profiles $H(\xi)$,

$$
H^{\prime \prime \prime}=\frac{\delta}{H^{2}+H},
$$

where we introduced a reduced capillary number, 


$$
\delta=3 \mathrm{Ca} / \theta_{e q}^{3} .
$$

We anticipate that $\delta$ will turn out small. At distances much larger than the slip length, the equation reduces further to

$$
y^{\prime \prime \prime}=\frac{1}{y^{2}},
$$

where we have put $H(\xi)=\delta^{1 / 3} y(\xi)$. This equation has an exact solution, whose properties have been summarized in [18]. In parametric form, the solution with a contact line $y(0)=0$ reads

$$
\left.\begin{array}{l}
\xi=\frac{2^{1 / 3} \pi \operatorname{Ai}(s)}{\beta[\alpha \operatorname{Ai}(s)+\beta \operatorname{Bi}(s)]} \\
y=\frac{1}{[\alpha \operatorname{Ai}(s)+\beta \operatorname{Bi}(s)]^{2}}
\end{array}\right\} s \in\left[s_{1}, \infty[,\right.
$$

where $\mathrm{Ai}$ and $\mathrm{Bi}$ are Airy functions [20]. The limit $\xi \rightarrow 0$ corresponds to $s \rightarrow \infty$, while the opposite limit $\xi \rightarrow \infty$ corresponds to $s \rightarrow s_{1}$, where $s_{1}$ is a root of the denominator of Eq. (14):

$$
\alpha \operatorname{Ai}\left(s_{1}\right)+\beta \operatorname{Bi}\left(s_{1}\right)=0 .
$$

Since the solution extends to $s=\infty, s_{1}$ has to be the largest root of Eq. (15).

The solution $y(\xi)$ is thus characterized by $\alpha, \beta$, and $s_{1}$, but only two of these parameters are independent due to Eq. (15). As detailed in [13], the constant $\beta$ can be determined by matching Eq. (14), which is valid only for $\xi \gtrsim 1$, to a solution of Eq. (11), which includes the effect of the cutoff and is thus valid down to the position $\xi=0$ of the contact line. It was found that

$$
\beta^{2}=\pi \exp [-1 /(3 \delta)] / 2^{2 / 3}+O(\delta),
$$

which eliminates one of the two free parameters. The remaining parameter will be eliminated below by matching $y(\xi)$ to the film solution.

The exact solutions presented by Duffy and Wilson [18] behave as $y \sim \xi^{2}$ for $\xi \rightarrow \infty$ with a positive curvature. Note that this asymptotics cannot be matched directly to a rim of negative curvature. As can be seen from the solid line in Fig. 2 , however, some of the exact solutions exhibit a regime where the interface displays a pronounced maximum $y_{\max }$ that can be identified with the rim. As such, the matching of the rim to the contact line is implicitly taken care of in the Duffy-Wilson solution. After this maximum, the shape develops a minimum $y_{\min }$ before the solution diverges as $y \sim \xi^{2}$ for $\xi \rightarrow \infty$. We will show below that the size of the rim, characterized by $y_{\max } / y_{\min }$, becomes arbitrary large in the limit that the parameter $s_{1}$ approaches the rightmost zero of the Airy function $s_{0}=-2.338 \ldots$. This is indicated in Fig. 3. We therefore introduce an expansion parameter

$$
\epsilon=s_{1}-s_{0},
$$

which will give a diverging ratio $y_{\max } / y_{\min } \sim(-\ln \epsilon)^{1 / 3} / \epsilon$ for small values of $\epsilon$. It will turn out that $y_{\max } / y_{\min } \sim w / h_{f}$, which corresponds precisely to the asymptotic limit we intend to analyze.

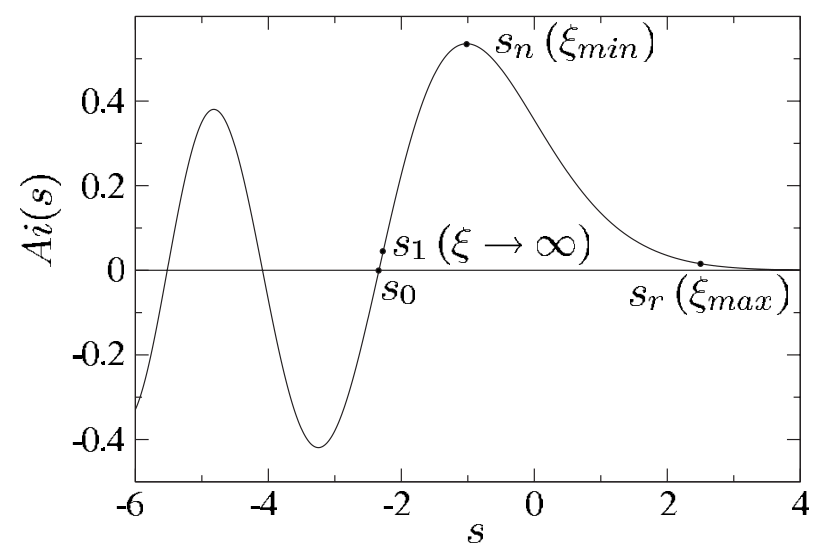

FIG. 3. The Airy function $\operatorname{Ai}(s)$ and the values of $s$ that are relevant in the analysis. The solution runs between $s=\infty$ (the contact line $\xi=0$ ) and $s=s_{1}$ (infinitely far from the contact line $\xi=\infty$ ). In particular, we define $s_{0}=-2.338 \ldots$ as the rightmost zero of Ai, and $s_{1}=s_{0}+\epsilon$. The rightmost maximum of $\mathrm{Ai}$ is defined as $s_{n}=$ $-1.088 \ldots$, which corresponds to the minimum at the neck behind the $\operatorname{rim}\left(\xi_{\text {min }}\right)$. The value $s_{r} \sim(-\ln \epsilon)^{2 / 3}$ corresponds to the maximum thickness of the rim $\left(\xi_{\text {max }}\right)$.

We now identify the relevant asymptotic properties of $y(\xi)$ for small values of $\epsilon$, for which it is convenient to introduce

$$
z(s)=\alpha \operatorname{Ai}(s)+\beta \operatorname{Bi}(s) .
$$

First, one can compute $\alpha$ from Eq. (15), i.e., $z\left(s_{1}\right)=0$, in the limit of small $\epsilon$ by expanding $\mathrm{Ai}(s)$ around $s_{0}$. Since $\operatorname{Ai}\left(s_{0}\right)$ $=0, \beta$ is of order $\epsilon$ and

$$
z\left(s_{1}\right)=\beta \operatorname{Bi}\left(s_{0}\right)+\epsilon \alpha \mathrm{Ai}^{\prime}\left(s_{0}\right)+O\left(\epsilon^{2}\right)=0 .
$$

Thus,

$$
\alpha=\frac{\beta}{c \epsilon}+O\left(\epsilon^{0}\right)
$$

where the constant is

$$
c=-\mathrm{Ai}^{\prime}\left(s_{0}\right) / \mathrm{Bi}\left(s_{0}\right) \approx-1.544710482 .
$$

Now since $\beta$ is known from Eq. (16), we have succeeded in computing the entire solution in terms of the single parameter $\epsilon$. Next we connect $\epsilon$ to the various geometrical properties of the rim.

Extrema of $y(\xi)$ correspond to $z^{\prime}(s)=0$, and can thus be found from solving

$$
\mathrm{Ai}^{\prime}(s)+c \epsilon \mathrm{Bi}^{\prime}(s)=0 .
$$

For small $\epsilon$, Eq. (21) has two zeros (cf. Fig. 3):

(i) one zero at $s_{r} \gg 1$, corresponding to the maximum of $y$ at the rim, denoted as $y_{\max }$ and

(ii) near $s_{n}$, defined by $\mathrm{Ai}^{\prime}\left(s_{n}\right)=0$, there is a minimum of $y$, which corresponds to the neck of thickness $y_{\min }$ behind the rim.

The value of $s_{r}$, which corresponds to the rim height, can be computed by expanding the Airy functions for large $s$ : 


$$
\begin{aligned}
& \operatorname{Ai}(s)=\frac{e^{-(2 / 3) s^{3 / 2}}}{2 \sqrt{\pi} s^{1 / 4}}\left(1+\frac{5}{48} s^{-3 / 2}+O\left(s^{-3}\right)\right), \\
& \operatorname{Bi}(s)=\frac{e^{(2 / 3) s^{3 / 2}}}{\sqrt{\pi} s^{1 / 4}}\left(1+\frac{5}{48} s^{-3 / 2}+O\left(s^{-3}\right)\right) .
\end{aligned}
$$

Thus, we find

$$
\epsilon=-\frac{\mathrm{Ai}^{\prime}\left(s_{r}\right)}{c \mathrm{Bi}^{\prime}\left(s_{r}\right)} \simeq \frac{1}{2 c} \exp \left(-\frac{4 s_{r}^{3 / 2}}{3}\right)\left(1+\frac{7 s_{r}^{-3 / 2}}{24}\right),
$$

or equivalently

$$
e^{-(4 / 3) s_{r}^{3 / 2}}=2 c \epsilon\left(1+\frac{7}{18 \ln (2 c \epsilon)}\right)
$$

which is the condition for the zero near the rim. The position of the maximum can now be determined from Eqs. (14) and (23):

$$
\begin{aligned}
\xi_{\text {max }} \simeq & \frac{2^{1 / 3} \pi}{\beta^{2}} e^{-(4 / 3) s_{r}^{3 / 2}}\left(1+\frac{s_{r}^{-3 / 2}}{24}\right) \simeq \frac{c \pi \epsilon}{2^{2 / 3} \beta^{2}}\left(1+\frac{1}{3 \ln (2 c \epsilon)}\right), \\
y_{\text {max }} \simeq & \frac{\pi s_{r}^{1 / 2}}{4 \beta^{2}} e^{-(4 / 3) s_{r}^{3 / 2}}\left(1+\frac{7 s_{r}^{-3 / 2}}{24}\right) \simeq \frac{c \pi \epsilon}{2 \beta^{2}}\left(-\frac{3}{4} \ln (2 c \epsilon)\right)^{1 / 3} \\
& \times\left\{1+O(\ln [2 c \epsilon])^{-2}\right\} .
\end{aligned}
$$

From Eq. (21) it follows that the zero corresponding to the neck is $s_{n}=-1.088 \ldots$ up to corrections of higher order in $\epsilon$. Inserting $s=s_{n}$ into Eq. (14), and using Eq. (19), we find

$$
\begin{gathered}
\xi_{\text {min }}=\frac{c 2^{1 / 3} \pi \epsilon}{\beta^{2}}+O\left(\epsilon^{2}\right), \\
y_{\text {min }} \simeq \frac{c^{2} \epsilon^{2}}{\beta^{2} \operatorname{Ai}\left(s_{n}\right)^{2}}+O\left(\epsilon^{3}\right) .
\end{gathered}
$$

These scalings imply that $y_{\max } / y_{\min } \sim(-\ln \epsilon)^{1 / 3} / \epsilon$, which indeed is asymptotically large.

An important observation is that in the limit of small $\epsilon$, $\xi_{\text {min }}=2 \xi_{\text {max }}$, showing that the large-scale structure of the rim is symmetric in this limit. One also verifies that the rim takes a parabolic shape,

$$
y(\xi) \simeq y_{\max }\left[1-\left(\frac{\xi-\xi_{\max }}{\xi_{\max }}\right)^{2}\right],
$$

for large values of $y$, i.e., away from $\xi=0$ and $\xi_{\text {min }}$. This corresponds to the equilibrium shape of an interface that is unaffected by viscous forces. However, note that owing to logarithmic corrections to $\xi_{\max }$ and $y_{\max }$, the approach to the equilibrium shape is quite slow. In Fig. 2, for example, deviations from a parabola remain quite pronounced. In addition, one recognizes that $\xi_{\text {min }}$ sets the width of the rim as

$$
\frac{w}{\lambda}=\frac{3 \xi_{\min }}{2 \theta_{e q}}=\frac{3 c \pi \epsilon}{2^{2 / 3} \theta_{e q} \beta^{2}} .
$$

We have based this definition on the total width of the rim rather than its half-width to avoid the logarithmic corrections in Eq. (25). These subtle differences will have a small effect on the definition of the apparent contact angle, as we will see below.

Upon approaching $\xi_{\text {min }}$, however, the solution develops a logarithmic dependence that is crucial for matching to the film region. To identify this "Voinov" behavior, we analyze the intermediate asymptotics for $y_{\min } \ll y(\xi) \ll y_{\max }$. In terms of $s$, this corresponds to $s_{n} \ll s \ll s_{r}$. It is convenient to introduce, consistent with Eqs. (27) and (28),

$$
\begin{gathered}
\Xi=\frac{\xi_{\min }-\xi}{\epsilon^{2}}, \\
Y=\frac{y}{\epsilon^{2}} .
\end{gathered}
$$

The scaling with $\epsilon^{2}$ ensures that $Y_{\min }$ becomes independent of $\epsilon$, while $Y_{\max }$ is pushed to infinity for small $\epsilon$. The intermediate asymptotics can thus be assessed by first taking the limit $\epsilon \rightarrow 0$ for finite $s$, and then consider large $s$. The leading-order expansion in $\epsilon$ becomes

$$
\begin{aligned}
\xi_{\text {min }}-\xi & =\frac{2^{1 / 3} \pi \operatorname{Ai}\left(s_{n}\right)}{\beta\left[\alpha \operatorname{Ai}\left(s_{n}\right)+\beta \operatorname{Bi}\left(s_{n}\right)\right]}-\frac{2^{1 / 3} \pi \operatorname{Ai}(s)}{\beta[\alpha \operatorname{Ai}(s)+\beta \operatorname{Bi}(s)]} \\
& =\frac{2^{1 / 3} \pi}{\alpha^{2}}\left(\frac{\operatorname{Bi}(s)}{\operatorname{Ai}(s)}-\frac{\operatorname{Bi}\left(s_{n}\right)}{\operatorname{Ai}\left(s_{n}\right)}+O(\epsilon)\right)
\end{aligned}
$$

and thus

$$
\Xi=\frac{2^{1 / 3} \pi c^{2}}{\beta^{2}}\left(\frac{\mathrm{Bi}(s)}{\operatorname{Ai}(s)}-\frac{\operatorname{Bi}\left(s_{n}\right)}{\operatorname{Ai}\left(s_{n}\right)}\right)+O(\epsilon) .
$$

Similarly, we have

$$
Y^{\prime}=-y^{\prime}=-\frac{2^{2 / 3} z^{\prime}(s)}{z(s)}=-\frac{2^{2 / 3} \mathrm{Ai}^{\prime}(s)}{\operatorname{Ai}(s)}+O(\epsilon) .
$$

Now we consider the limit of large $s$ :

$$
\begin{gathered}
\frac{Y^{\prime 3}}{3}=\frac{4}{3} s^{3 / 2}\left(1+\frac{3}{4 s^{3 / 2}}\right)=\frac{4}{3} s^{3 / 2}+1, \\
\Xi=\frac{2^{4 / 3} \pi c^{2}}{\beta^{2}} e^{(4 / 3) s^{3 / 2}} .
\end{gathered}
$$

In terms of the slope $Y^{\prime}(\Xi)$, this yields the Voinov scaling [21]

$$
Y^{\prime 3}=3 \ln \left(\frac{\beta^{2} e \Xi}{2^{4 / 3} \pi c^{2}}\right),
$$

where $e=\exp (1)$.

\section{B. Film solution}

We now show how the back of the rim connects to the film, which is at around $x_{0} \approx 2 w$. This crossover region, including the film, is described by Eq. (8) for which $h_{f}$ provides the length scale. We therefore analyze the back of the rim by introducing another similarity function 


$$
h(x)=h_{f} G\left(\frac{\left(x-x_{0}\right) \mathrm{Ca}^{1 / 3}}{h_{f}}\right), \quad \zeta=\frac{\left(x-x_{0}\right) \mathrm{Ca}^{1 / 3}}{h_{f}},
$$

which transforms Eq. (8) into the famous Landau-Levich equation [22]

$$
G^{\prime \prime \prime}=\frac{3}{G^{2}}\left(1-\frac{1}{G}\right) .
$$

The boundary condition for our problem reads $G \rightarrow 1$ as $\zeta$ $\rightarrow \infty$. This solution was previously analyzed by Tuck and Schwartz [23] and below we closely follow their analysis.

For $\zeta \rightarrow-\infty$, solutions of Eq. (39) generally grow quadratically [24]. To match to the Voinov solution (37), we are interested in solutions which only grow linearly (with logarithmic corrections). These solutions are characterized by the fact that the curvature vanishes at infinity. This eliminates one degree of freedom and gives

$$
G^{\prime 3} \simeq-9 \ln \left(a\left[\zeta_{0}-\zeta\right]\right),
$$

for $\zeta \rightarrow-\infty$. The parameter $\zeta_{0}$ is the remaining degree of freedom and reflects the translational invariance of the solution.

The asymptotic behavior (40) has to be compared to Eq. (37). Therefore, our main interest is to determine the constant $a$ inside the logarithm, which has to be determined by solving Eq. (39) numerically. Note that Tuck and Schwartz [23] numerically solved for $G(\zeta)$, but did not report the value of $a$. We do this following standard procedure [23]: "shooting" from the film to the negative $\zeta$ direction and adjusting a constant so as to satisfy the boundary condition for $\zeta \rightarrow-\infty$. Linearizing around the film $G=1$ using

$$
G=1+\varepsilon e^{\sigma \zeta},
$$

one finds three eigenvalues: $\sigma=3^{1 / 3},-3^{1 / 3}(1+i) / 2$, and $-3^{1 / 3}(1-i) / 2$. We are only interested in the two eigenvalues with negative real part, which grow as $\zeta$ becomes more negative. The third decays as one shoots from inside the film.

Because of translational invariance, one of the two remaining degrees of freedom can be absorbed into a phase factor, so the initial condition for the shooting procedure becomes

$$
G=1+\varepsilon e^{-3^{1 / 3} \xi / 2} \cos \left(3^{1 / 3} \zeta / 2\right),
$$

where $\varepsilon$ is the only free parameter. Using Eq. (42), Eq. (39) is integrated backward to large negative values of $\zeta$. The amplitude $\varepsilon$ is adjusted, so that the curvature goes to zero as $\zeta \rightarrow-\infty$, fixing the solution uniquely. The resulting profile is shown in Fig. 4. Comparing the asymptotics of this solution to Eq. (40), we find $a=1.094 \ldots$.

\section{Matching}

We now match the logarithmic variations of the slope observed for the solutions containing the contact line and film, respectively. In original variables Eqs. (37) and (40) become

$$
h_{c l}^{\prime 3}=-9 \mathrm{Ca} \ln \left(\frac{\beta^{2} e \theta_{e q}\left(x_{\min }-x\right)}{3 \times 2^{4 / 3} \pi c^{2} \epsilon^{2} \lambda}\right),
$$

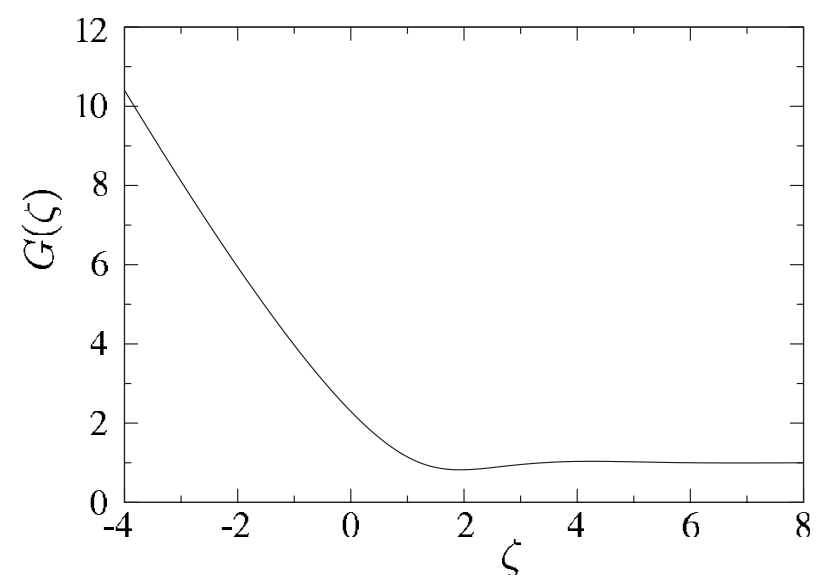

FIG. 4. The similarity solution $G(\zeta)$ near the flat film that has to be matched to the rim. This curve was also computed in [23].

$$
h_{f}^{\prime 3}=-9 \mathrm{Ca} \ln \left(\frac{a \mathrm{Ca}^{1 / 3}\left(x_{0}-x\right)}{h_{f}}\right),
$$

so the two solutions indeed match. Apart from the trivial $x_{0}=x_{\min }$, this gives the matching condition

$$
\frac{a \mathrm{Ca}^{1 / 3}}{h_{f}}=\frac{\theta_{e q} \beta^{2} e}{3 \times 2^{4 / 3} \pi c^{2} \epsilon^{2} \lambda} .
$$

For given values of $h_{f}$ and $\lambda$, this equation contains three unknown parameters, namely, $\epsilon, \beta$, and the capillary number Ca. Eliminating $\epsilon$ between Eqs. (45) and (30), we find

$$
\beta^{2}=\frac{3 e h_{f} \lambda \pi}{2^{8 / 3} a \mathrm{Ca}^{1 / 3} w^{2} \theta_{e q}} .
$$

Using Eqs. (16) and (12) this gives the central result of the paper:

$$
\mathrm{Ca}=\frac{\theta_{e q}^{3}}{9}\left[\ln \left(\frac{4}{3 e} a \theta_{e q} \mathrm{Ca}^{1 / 3} \frac{w^{2}}{\lambda h_{f}}\right)\right]^{-1} .
$$

This equation determines the speed of a dewetting contact line with equilibrium angle $\theta_{e q}$, for given values of $\lambda, h_{f}$, and $w$. The dependence of the speed on the cube of the equilibrium angle, characteristic for all wetting problems, has been predicted in $[14,15]$. For completeness, we express the expansion parameter $\epsilon$ in terms of the physical parameters of the problem, by multiplying Eqs. (30) and (45):

$$
\epsilon=\frac{e}{4 a c \mathrm{Ca}^{1 / 3}} \frac{h_{f}}{w} .
$$

\section{Numerics}

To test the accuracy of our predictions, we performed numerical simulations of the fully time-dependent lubrication equation (1). We use a finite difference scheme very similar to that employed in [25], splitting Eq. (1) into two lowerorder equations

$$
\partial_{t} h=U \partial_{x} h-\partial_{x} v,
$$




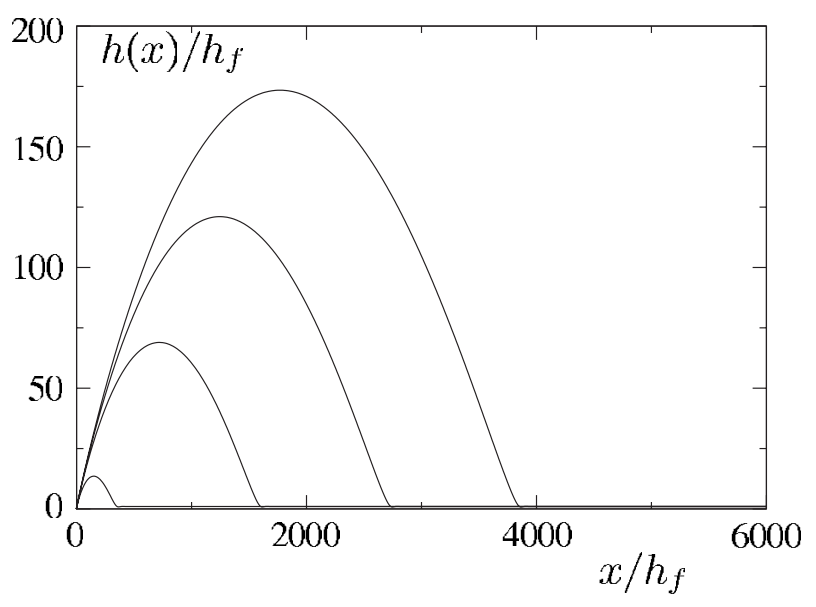

FIG. 5. A numerical simulation of Eq. (1), showing the growing rim. The frame of reference is chosen such that the tip of the receding front remains at the origin. The slip length is $\lambda=10^{-4} h_{f}$ and $\theta_{e q}=0.3$.

$$
v=\frac{\gamma}{3 \eta}\left[h^{2}(h+3 \lambda) \partial_{x x x} h\right] .
$$

The nodes for the velocity $v$ are defined at the midpoint between two nodes for the profile $h$. At the end of the liquid film we use a local expansion of the profile on the basis of the leading balance (7), which gives

$$
h(x)=\theta_{e q} x+\left(a_{2}+\frac{\mathrm{Ca}}{2 \theta_{e q} \lambda} \ln x\right) x^{2} .
$$

Here, $a_{2}$ is a free parameter used to interpolate the profile. At the right the profile is held at a fixed value $h_{f}$, corresponding to the film thickness. Both at the end of the film and in the neck region, our grid is highly refined to ensure that the highest derivatives are properly represented.

A typical result of a simulation run is shown in Fig. 5. Owing to our choice of reference frame, the rim remains in place, but grows slowly according to estimate (5). The speed of retraction is recovered from Eq. (50). The slip length is chosen to be smaller than $h_{f}$ by a factor of $10^{-4}$. The rim width is allowed to grow to more than 1000 times the film thickness. In Fig. 6, the speed of retraction is plotted against the increasing rim width; it decreases logarithmically, as predicted by Eq. (46). The inset demonstrates the remarkable agreement between simulation (full line) and theory (dashed line). This is possible only because all the numerical factors inside the logarithm have been captured.

\section{DISCUSSION}

Previous analyses $[1,16]$ have considered the present problem from the point of view of dynamic contact angles. The idea is to equate the dynamic contact angles on both sides of the rim (advancing and receding). To obtain an explicit prediction for the speed, one needs to close to problem by a relation between the contact angle and speed. It is interesting to review this interpretation from the perspective of our matched asymptotic analysis.

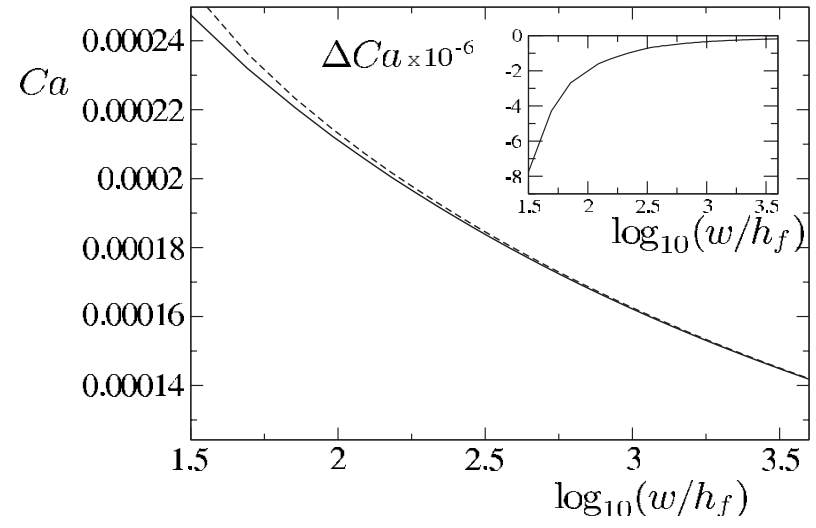

FIG. 6. A numerical test of our theoretical prediction (46) for the speed of retraction, using the simulation shown in Fig. 5. As the rim width $w$ increases, the speed decreases. The full line comes from the numerical simulation of Eqs. (48) and (49), while the dashed line represents Eq. (46). The inset shows the difference between theory and simulation.

At large scales, the rim takes the equilibrium shape of a parabola with an apparent contact angle $\theta_{a p}$ :

$$
h=h_{r}\left[1-\left(\frac{x-w}{w}\right)^{2}\right] \text {. }
$$

The angle at which this parabola intersects with the substrate is defined as the apparent contact angle

$$
\theta_{a p}=2 h_{r} / w .
$$

Using rescalings (10) and (12), one thus finds that

$$
\theta_{a p}=4 \delta^{1 / 3} \theta_{e q} \frac{y_{\max }}{\xi_{\min }},
$$

remembering that our definition of $w$ is based on half the total width of the rim. Inserting expressions (26) and (27), we find

$$
\theta_{a p}^{3}=-9 \mathrm{Ca} \ln (2 c \epsilon) .
$$

Note that had $\theta_{a p}$ been based on the half-width of the rim as defined by the position $\xi_{\max }$ of the maximum, the result would have been

$$
\widetilde{\theta}_{a p}^{3}=-9 \mathrm{Ca} \ln \left(\frac{2 c \epsilon}{e}\right),
$$

since $\xi_{\max }$ has its own logarithmic correction. This is a subtlety absent, for example, from the analysis of a spreading drop [26].

Now using Eqs. (46) and (47) one finds that

$$
\ln (2 c \epsilon)=\ln \frac{2 \theta_{e q} w}{3 \lambda}-\frac{\theta_{e q}^{3}}{9 \mathrm{Ca}},
$$

and thus 


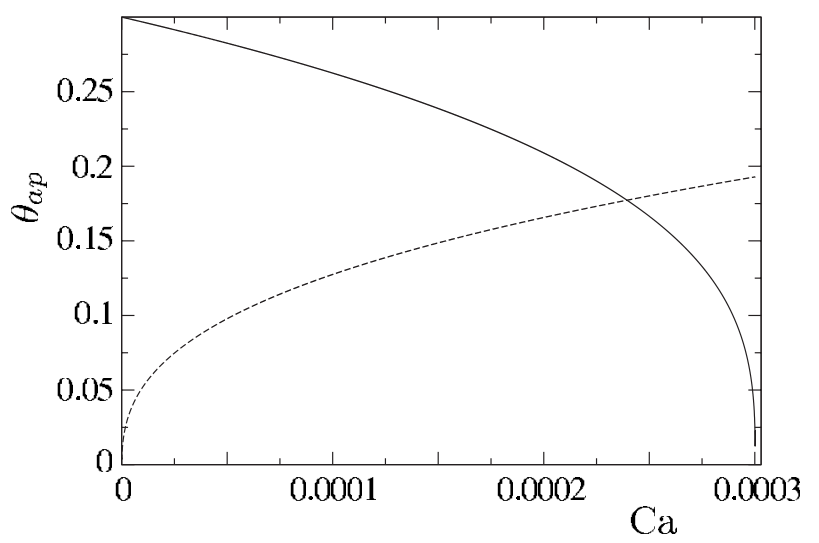

FIG. 7. Apparent contact angle $\theta_{a p}$ for the receding and advancing fronts (solid and dashed lines, respectively) for $\theta_{e q}=0.3$. Length scales were chosen as $\lambda=10^{-9} \mathrm{~m}, h_{f}=10^{-6} \mathrm{~m}$, and $w=10^{-4} \mathrm{~m}$. The intersection of the curves provides the selection of angle and speed.

$$
\theta_{a p}^{3}=\theta_{e q}^{3}-9 \mathrm{Ca} \ln \frac{2 \theta_{e q} w}{3 \lambda},
$$

which can be interpreted as the Cox-Voinov relation for the receding contact line $[2,21,27]$, with $\lambda$ and $w$ as the inner and outer length scales. The analysis of [16], however, does not permit us to calculate the prefactors inside the logarithm, but merely identifies the argument inside the logarithm as a ratio of $w / \lambda$.

Similarly, using Eqs. (52) and (47), one derives an alternative expression for $\theta_{a p}$ :

$$
\theta_{a p}^{3}=9 \mathrm{Ca} \ln \frac{2 a \mathrm{Ca}^{1 / 3} w}{e h_{f}},
$$

which contains the characteristic scales $h_{f}$ and $w$. Note that the prefactors inside the logarithm are not universal and originate from the details of the matching. As usual for a vanishing contact angle [28], there appears a speeddependent factor inside the logarithm, which is $\mathrm{Ca}^{1 / 3}$ in our case, as derived from the scale transformation (38).

Equating the advancing and receding angles indeed selects the dewetting velocity (46). This is illustrated in Fig. 7, where we plot the apparent contact angles for $\theta_{e q}=0.3$ and representative values for the length scales (see caption). For large rims, the resulting apparent angle can be approximated as

$$
\frac{\theta_{a p}}{\theta_{e q}} \simeq\left(1+\frac{\ln \frac{2 \theta_{e q} w}{3 \lambda}}{\ln \frac{2 e a \mathrm{Ca}^{1 / 3} w}{h_{f}}}\right)^{-1 / 3} .
$$

This result can be interpreted as a competition between the dissipation in the advancing part, tending to increase the angle, and the receding part, tending to lower the angle. Note that this result is manifestly different from the analysis of $[1,15]$, which incorrectly predicts a power of $1 / 2$ instead of $1 / 3$ in Eq. (56). This can be traced back to the approximation used by $[1,15]$ for the evaluation of the energy dissipation, which only holds in the linear regime, where $\theta_{a p}$ is close to $\theta_{e q}[2]$.

On the basis of this simplified dissipation argument [1], arrive at the approximation $\theta_{a p} \approx \theta_{e q} / 4$ for the apparent contact angle. However, this result contradicts the analysis of the stability of a receding wetting line, performed on the basis of the same theory [17], which predicts instability for angles $\theta_{a p}<\theta_{e q} / \sqrt{3}$. In other words, the rim would not recede leaving behind a dry solid, but rather would once again leave a film. We stress that this inconsistency is not the result of the principles used in its derivation, but simply results from an inadequate approximation for the dissipation taking place close to the contact line.

Finally, we comment on the experimental situation, which is unsatisfactory at present. Experiments on macroscopic films were performed by [12] and were taken up again by $[11,29]$. The dependence of the speed of retraction on the cube of the equilibrium contact angle was confirmed by [12]. Measurements of the apparent contact angle were performed by [12], giving $\theta_{a p} \approx 0.7 \theta_{e}$. On the other hand, detailed measurements based on the method of refraction of a mesh underneath the film gave much smaller angles, closer to $\theta_{a p}$ $\approx 0.15 \theta_{e}$. In neither case it was made clear where exactly the angle was measured. The only way of finding a unique angle, consistent with theory, is to fit a section of a circle to the rim, and then to determine the angle of intersection with the substrate. To the best of our knowledge, a measurement of this type remains to be done.

\section{ACKNOWLEDGMENTS}

This research was conceived and performed during a visit to HKUST. We gratefully acknowledge the kind hospitality of the Physics and Mathematics Departments there, and in particular Ping Sheng, Xiao-Ping Wang, and Thiezheng Qian.
[1] P.-G. de Gennes, F. Brochart-Wyart, and D. Quéré, Capillarity and Wetting Phenomena: Drops, Bubbles, Pearls, Waves (Springer, New York, 2003).

[2] D. Bonn, J. Eggers, J. Indekeu, J. Meunier, and E. Rolley, Rev. Mod. Phys. 81, 739 (2009).

[3] G. Reiter, Phys. Rev. Lett. 68, 75 (1992).

[4] F. Saulnier, E. Raphaël, and P.-G. de Gennes, Phys. Rev. Lett.
88, 196101 (2002).

[5] R. Seemann, S. Herminghaus, C. Neto, S. Schlagowski, D. Podzimek, R. Konrad, H. Mantz, and K. Jacobs, J. Phys.: Condens. Matter 17, S267 (2005).

[6] J. Buehrle, S. Herminghaus, and F. Mugele, Phys. Rev. Lett. 91, 086101 (2003).

[7] R. Seemann, S. Herminghaus, and K. Jacobs, J. Phys.: Con- 
dens. Matter 13, 4925 (2001).

[8] R. Fetzer, K. Jacobs, A. Münch, B. Wagner, and T. P. Witelski, Phys. Rev. Lett. 95, 127801 (2005).

[9] F. Saulnier, E. Raphaël, and P.-G. de Gennes, Phys. Rev. E 66, 061607 (2002).

[10] S. Herminghaus, R. Seemann, and K. Jacobs, Phys. Rev. Lett. 89, 056101 (2002).

[11] C. Andrieu, Ph.D. thesis, Université Paris VI, 1995.

[12] C. Redon, F. Brochard-Wyart, and F. Rondelez, Phys. Rev. Lett. 66, 715 (1991).

[13] J. Eggers, Phys. Fluids 17, 082106 (2005).

[14] P.-G. de Gennes, C. R. Acad. Sci., Ser. II: Mec., Phys., Chim., Sci. Terre Univers 303, 1275 (1986).

[15] F. Brochard-Wyart, J.-M. di Meglio, and D. Quéré, C. R. Acad. Sci., Ser. II: Mec., Phys., Chim., Sci. Terre Univers 304, 553 (1987).

[16] J. C. Flitton and J. R. King, J. Eng. Math. 50, 241 (2004).

[17] P.-G. de Gennes, Colloid Polym. Sci. 264, 463 (1986).

[18] B. R. Duffy and S. K. Wilson, Appl. Math. Lett. 10, 63 (1997).
[19] C. Huh and L. E. Scriven, J. Colloid Interface Sci. 35, 85 (1971)

[20] M. Abramowitz and I. A. Stegun, Handbook of Mathematical Functions (Dover, New York, 1968).

[21] O. V. Voinov, Fluid Dyn. 11, 714 (1976).

[22] L. D. Landau and B. V. Levich, Acta Physicochim. URSS 17, 42 (1942).

[23] E. O. Tuck and L. W. Schwartz, SIAM Rev. 32, 453 (1990).

[24] C. M. Bender and S. A. Orszag, Advanced Mathematical Methods for Scientists and Engineers (McGraw-Hill, New York, 1978).

[25] J. Eggers and T. F. Dupont, J. Fluid Mech. 262, 205 (1994).

[26] L. M. Hocking, Q. J. Mech. Appl. Math. 36, 55 (1983).

[27] R. G. Cox, J. Fluid Mech. 168, 169 (1986).

[28] J. Eggers and H. A. Stone, J. Fluid Mech. 505, 309 (2004).

[29] C. Andrieu, C. Sykes, and F. Brochard, J. Adhes. 58, 15 (1996). 